\title{
Brain Imaging and Aging
}

\author{
Shimon Shatzmiller*, Galina M Zats and Inbal Lapidot \\ Department of Biological Chemistry, Ariel University, Israel \\ *Corresponding Author: Shimon Shatzmiller, Department of Biological \\ Chemistry, Ariel University, Israel.
}

DOI: 10.31080/ASNE.2020.03.0209
Received: July 03, 2020

Published: July 30, 2020

(C) All rights are reserved by Shimon

Shatzmiller., et al.
Brain imaging is one of the main obstacles of modern disease diagnostics since it has to allow accurate and quantitative measurements on a living organ that is placed in adults (in neonatal humans skull penetration is somewhat easier) inside a bone cage. Inside is the brain, the main function controller of our body that has to be done in a non-invasive way on the living operation brain. Many spectroscopic methods are trying to overcome the difficult access to the brain: Photoacoustic microscopy, Confocal microscopy, Two-photon microscopy, Optical coherence tomography, Scanning Laser Acoustic Microscopy, Acoustic microscopy, Ultrasonography, positron tomography, fluorescence methods, photo caustic microscopy and multy (two) photon imaging spectroscopy. The situation is complex since the brain is wrapped in the blood brain barrier allowing only selected molecules to pass from the blood stream to and out of the brain. In this chapter, we will survey the current situation of brain diagnostics with the aid of the spectroscopic methods. Brain research is integrated in aging research as a major area of interest. Aging is in most cased coupled with the loss of brain function and dementia. The neurodegenerative diseases, although identified by Dr. Alzheimer and his collaborators more than a century ago, continue to be the leading causes of mortality among the elderly. Brain research in trying to give hope to those people but unfortunately our understanding in this area is limited.

\section{Opening remarks and some instrumental methods}

Imaging is becoming an increasingly important tool in both research and clinical care. A range of imaging technologies now provide unprecedented sensitivity to visualization of brain structure and function from the level of individual molecules to the whole brain. Many imaging methods are noninvasive and allow dynamic processes to be monitored over time. Imaging is enabling researchers to identify neural networks involved in cognitive processes; understand disease pathways; recognize and diagnose diseases early, when they are most effectively treated; and determine how therapies work. Brain research is integrated in aging research as a major area of interest. Aging is in most cased coupled with the loss of brain function and dimensia. Researchers look for methods to quantitatively measure the following parameters that could as accurately as possible measure four different components in human brain studies:

- $\quad$ The volumes and positions of the different brain areas.

- The interconnections between different brain areas.

- The activity of a brain areas.

- The chemical composition of a brain areas.

Aging is very often accompanied by the appearance of brain dysfunction. In case of Brain Disorders, imaging test aid in the diagnosis. Your doctor will likely perform a neurological exam to check your vision, hearing, and balance. Your doctor might also get images of your brain to make a diagnosis. The most common healthcare facility common diagnostic imaging tools are CT, MRI (MRI DTI), and PET scans. Other methods, ultrasound imaging.

CT (Medical Computed Tomography) CT scan uses computerized combinations of many x-rays taken from different angles to produce cross-sectional (tomographic) (virtual slices) of specific scanned areas, allowing cross-sectional images (tomography). The user can see inside the object without cutting. 
Digital geometry processing is used to create a 3D image of the object's interior from a large series of two-dimensional radiographic images taken around a single axis of rotation.

Medical imaging is the most common application of CT x-ray. Its latitudinal images are used for diagnostic and therapeutic purposes in various medical fields. The remainder of this article discusses medical imaging of medical imaging; Industrial X- ray CT applications are discussed in industrial computed tomography scans.

MRI Magnetic Resonance Imaging (MRI) is a medical imaging technique used in radiology to create images of the anatomy and physiological processes of the body in both health and disease. MRI scanners use powerful magnetic fields, radio waves and field transitions to create images of the body's interior. For this technique, the patient is placed on a movable bed and placed in a strong magnetic field. The magnetic field causes parts of the brain to produce certain signals, which are then discovered and used by a computer to create images of everything in the brain. At best, this technique can visualize small structures up to half the diameter of human hair. Using these images researchers can measure the location or volume of each part of the brain, and then these measurements can be compared for people with ASD and neurotypical people. Diffusion-weighted magnetic resonance imaging (DWI or DW-MRI) is an imaging method that uses water molecules to disperse contrast MR images. This allows mapping of the diffusion process of molecules, especially water, in biological tissues, in vivo rather than invasively.

Molecular diffusion in tissues is not free, but reflects interactions with many obstacles, such as macromolecules, fibers and membranes. Water molecule diffusion patterns can therefore reveal microscopic details about tissue architecture, normalization or in a diseased state. A special type of DWI, Diffusion Tensor Imaging (DTI), is widely used to map white matter mask in the brain.

PET positron emission tomography is nuclear medicine, a functional imaging technique used to view metabolic processes in the body. The system detects gamma-ray pairs that are indirectly emitted by a radionuclide (portrone) emitting positron, which is inserted into the body on a biologically active molecule. Threedimensional images of the concentration of traces in the body are then constructed by computer analysis. In modern PET-CT scans, $3 \mathrm{D}$ imaging is usually performed with a CT scan performed on the patient at the same session, on the same machine.

If the biologically active molecule selected for PET is Pleodoxyglucose (FDG), analogous to glucose, the concentrations of the trace character will indicate tissue metabolic activity as it corresponds to regional glucose uptake. Use this router to explore possibility of cancer.
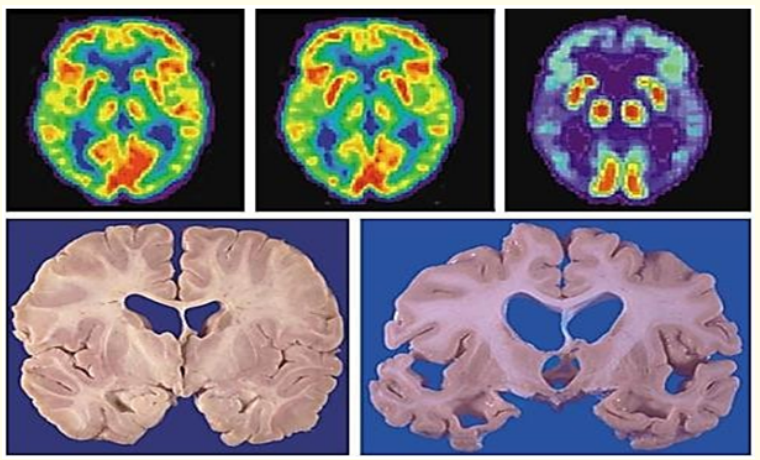

Figure 1

Alzheimer's disease causes functional and structural changes in the brain. Positron-emission tomographic (PET) scans of brains from a 20-year-old (top row, left), a normal 80-year-old (middle) and an Alzheimer's patient (right) show that the diseased brain has reduced blood volume change during the scan, indicating a decline in cognitive function. Coronal sections of postmortem brains illustrate normal morphology (left) and dramatic shrinkage in advanced Alzheimer's disease (right).

(PAT) Laser-induced 3D acoustic tomography, also called optoacoustic tomography, is being developed to visualize non-invasive animal brain structures when the skin and skull are intact. This mode of imaging combines the advantages of optical contrast and sonic resolution.

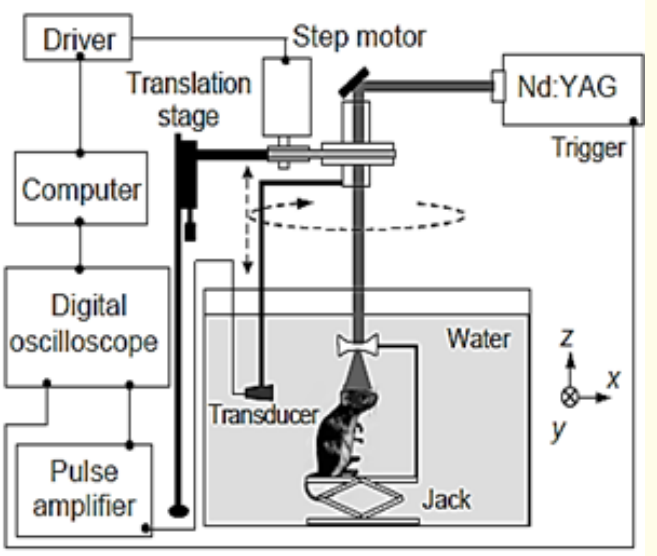

Figure 2: Setup for non-invasive transdermal and transcranial 3-D PAT of mouse brain. 
Photoacoustic tomography, a technique for imaging optical properties in biological tissue non-invasively, combines the benefits of both light and ultrasound.

Photoacoustic imaging (optical-acoustic imaging) is a biomedical imaging method based on the photoacoustic effect. In photoacoustic imaging, non-ionizing laser pulses are transmitted to biological tissues (when using radio frequency pulses, the technology is called thermo-acoustic imaging). Some of the transmitted energy will be absorbed and converted to heat, leading to transient thermoelastic expansion and thus broad ultrasonic emission (i.e. MHz). The generated sound waves are detected by sound transducers and then analyzed to produce images. Optical absorption is known to be closely related to physiological properties. As a result, the sound emission intensity (i.e., the photoacoustic signal), which is proportional to the local energy extract, reveals specific optical absorption contrast. You can then create 2D or 3D images of the targeted areas. Figure 2 is a schematic illustration showing the basic principles of photoacoustic imaging.

All these electronics based methods are applied virtual slices of the organ analyzed and signals are processed with computer management, and use "contrast chemical agents" to increase the respective electronic signals from the scans.

Other methods

\begin{tabular}{|c|c|c|c|c|c|c|}
\hline & $\Delta f$ & Primary contrast & $\Delta \mathrm{z}$ & $\delta z$ & $\delta x$ & Speed \\
\hline & $\mathrm{Hz}$ & & $\mathrm{mm}$ & $\mu m$ & $\mu m$ & $M v x / s$ \\
\hline Photoacoustic microscopy & $50 \mathrm{M}$ & Optical absorption & 3 & 15 & 45 & 0.5 \\
\hline Photoacoustic tomography & $5 \mathrm{M}$ & Optical absorption & 50 & 700 & 700 & 0.5 \\
\hline Confocal microscopy & & $\begin{array}{r}\text { Fluorescence, } \\
\text { scattering }\end{array}$ & 0.2 & $3-20$ & $0.3-3$ & $10-100$ \\
\hline Two-photon microscopy & & Fluorescence & $\begin{array}{r}0.5- \\
1.0\end{array}$ & $1-10$ & $0.3-3$ & $10-100$ \\
\hline Optical coherence tomography & $\begin{array}{r}300 \\
T\end{array}$ & Optical scattering & $1-2$ & $\begin{array}{r}0.5- \\
10\end{array}$ & $1-10$ & $\begin{array}{r}20- \\
4.000\end{array}$ \\
\hline $\begin{array}{l}\text { Scanning Laser Acoustic } \\
\text { Microscopy }\end{array}$ & $\begin{array}{r}300 \\
M\end{array}$ & Ultrasonic scattering & $1-2$ & 20 & 20 & 10 \\
\hline Acoustic microscopy & $50 \mathrm{M}$ & Ultrasonic scattering & 20 & $\begin{array}{l}20- \\
100\end{array}$ & $\begin{array}{r}80- \\
160\end{array}$ & 0.1 \\
\hline Ultrasonography & $5 \mathrm{M}$ & Ultrasonic scattering & 60 & 300 & 300 & 1 \\
\hline
\end{tabular}

Figure 3

Photoacoustic Imaging In biomedical, photoacoustic imaging is an emerging situation that greatly overcomes the resolution and depth limits of optical imaging while maintaining a relatively high contrast. Photoacoustic imaging (optical-acoustic imaging) is a biomedical imaging method based on the photoacoustic effect. In photo-acoustic imaging, non-ionizing laser sections are transferred to biological tissues (when using radio frequency pulses, the technology is called thermo-acoustic imaging). Some of the transmitted energy will be absorbed and converted to heat, leading to transient thermoelastic expansion and thus broad emission (i.e. MHz). The generated sound waves are detected by sound transducers and then analyzed to produce images. Optical absorption is known to be closely related to physiological properties, such as hemoglobin concentration and oxygen saturation. As a result, the sound emission intensity (i.e. the photoacoustic signal), which is proportional to the local energy extract, reveals specific optical absorption contrast. You can then create 2D or 3D images of the targeted areas. Figure 2 is a schematic illustration showing the basic principles of photo-acoustic imaging. A firm diagnosis of Alzheimer's disease (or most other advanced dementia) may take a long time. This is an elimination process, and there are literally hundreds of things that need to be considered as possible causes. The various tests are done (should) be done in succession, partly based on the probability that they will show something, partly on how easy it is to coordinate the test and partly on cost. PET scanning, for example, costs several thousand dollars, so it is not the first test done!.

The diagnosis is based on the radioactive glucose that has F-18 in its structure. Fluorine- $18\left(18^{\mathrm{F}}\right)$ is a fluorine radioisotope that is a major source of positrons. It has a mass of 18.0009380 (6) $u$ and its half life is 109.771 minutes. This is broken down by positron emission $97 \%$ of the time and electron capture $3 \%$ of the time. Both rotational states yield stable 18 oxygen. It is a short life and must be prepared in a nearby isotope chemical laboratory for brain PET scanning (Figure 4).

Toda, the synthesis may proceed with molecular fluorine-18.

In the effort to investigate the correlation between aging and $\mathrm{AD}$, it is established that [F-18] F DDNP micro PET imaging correlates with brain $\mathrm{A} \beta$ burden in a transgenic rat model of Alzheimer disease. Today, Complete diagnosis of Alzheimer's disease requires confirmation of diagnosis through a brain biopsy or autopsy. So your doctor's diagnosis is "reasonable" and not "absolute". 


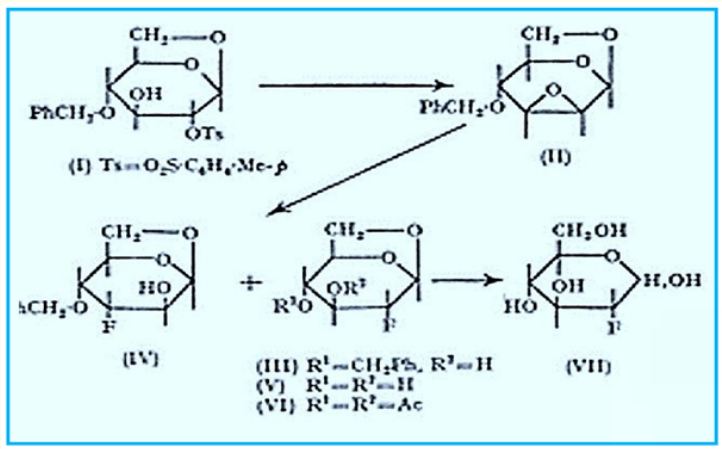

Figure 4: The first synthesis of fluorinated $18 \mathrm{~F}$ glucose.
Alzheimer's disease (AD) remains the only leading cause of death for which no disease- modifying treatment exists, and age is by far the greatest risk factor.

It is now estimated that $1 / 3$ of older individuals in the U.S. will die with dementia, Mainly because of the count. Our findings, which coincide with reports from autopsy studies, CSF (CSF) and other PET imaging studies (positron emission tomography), suggest a similar rate of $1 / 3$ of older people Proper (CN) express evidence. Of amyloid $B$ accumulation $(A \beta)$, one of the marked pathologies of $\mathrm{AD}$. Although people tend to shift the focus from amyloids to microbiome, and inflammation as neurodegenerative disorders, the amyloid beta polypeptide is found in damaged brain tissues, along with glutathione.

Alzheimer's disease represents one of the great unsolved medical needs confronting society during this millennium. Despite considerable work during the past quarter century. No medicines exist that attack the underlying pathophysiology of the disease. The situation is so unclear, that there is a conflict weather b-0amyloid peptides or glutathione should be regarded as biomarkers for AD. A biomarker is a measurable feature that can be used to predict a biological state. Examples of biomarkers might include levels of proteins in the blood, patterns of activity in the brain, or the amount of time it takes for an individual to complete a task. One of the cardinal features of $\mathrm{AD}$ is deposition of plaques comprised of aggregated (3-Amyloid peptides, $A \beta s$ ) in the brain. particularly in regions associated with cognition and memory Overproduction of $A \beta$ which appears to be directly neurotoxic. can be detected at the earliest stages of $\mathrm{AD}$ and. in fact. before cognitive dysfunction is detectable $\mathrm{Ab}$ is produced from its precursor protein. Amyloid precursor protein (APP). by proteolytic processing at its $\mathrm{N}$ - and Ctermini by $\beta$ - and $\gamma$-secretase enzymes respectively.

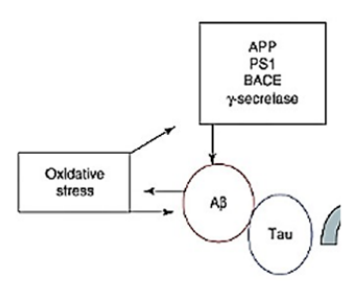

Oxidative stress early stages

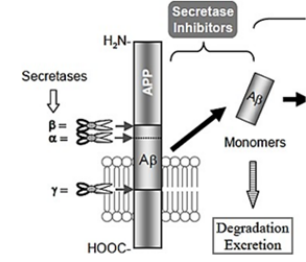

Amyloid hypothesis earlystages

\section{Figure 5}

Two main hypotheses are today directing neurodegenerative diseases research. The Amyloid hypothesis and the oxidative stress mechanism. Although accumulation of data supports the Amyloid beta aggregation hypothesis, in conclusion, the oxidative stress hypothesis of $\mathrm{AD}$ is still very much alive and viable, but a great deal of work needs to be done to design future studies and appropriate clinical trials that will conclusively establish the role of oxidative stress in $\mathrm{AD}$ pathogenesis.

One high hurdle is the lack of a quantitative instrumental method to diagnose and follow the disease from early stages. In particular the development of new drugs depends a lot of such an instrumental devise.

Alzheimer's Disease is one of the many neurodegenerative disorders that are tormenting many, in particular elderly people, and is in the unflavored situation where many theories on what initiates the cascade of events and when exactly it started to affect the life of the ill people. Today, only a post mortem autopsy and tissue analysis of the patient brain can diagnose $100 \%$ the Alzheimer's disease.

While most $\mathrm{AD}$ related pathology occurs in the brain, the disease has also been reported to affect the eye, which is more useful for imaging than the brain. AD-related proteins exist in the normal human eye and may produce ocular pathology in AD. There is some homology between the retinal and cerebral vasculatures and the retina also contains nerve cells and fibers that form a sensory ex- 
tension of the brain. The eye is the only location in the body where presents a review of current literature on ocular morphology in AD and discusses the potential for an ocular based screening test for AD.

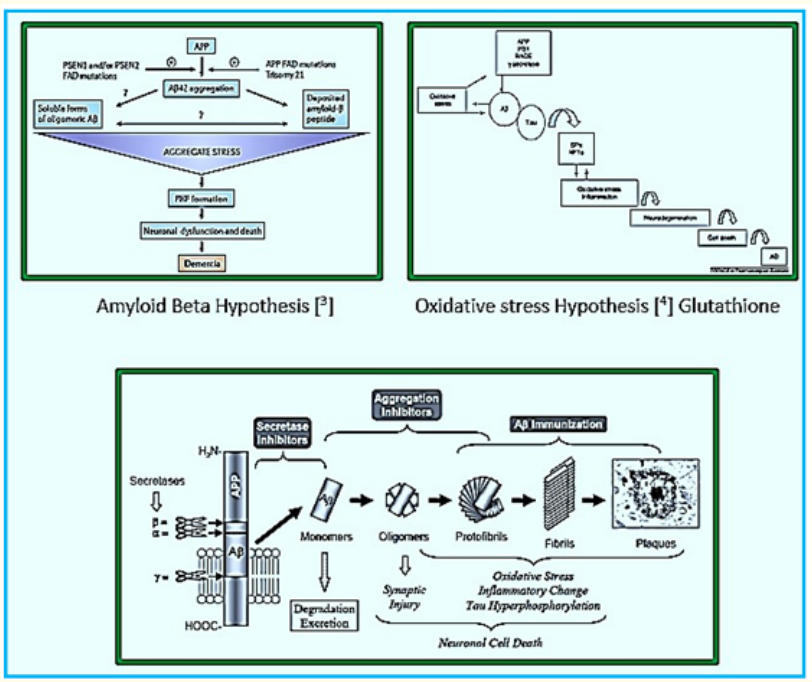

Figure 6

Schematic illustration of the A $\beta$ amyloid cascade from APP cleavage by secretases to generate $A \beta$ monomers, to plaque formation, via oligomers, protofibrils, and fibrils. Causative factors for neuronal injury are indicated in italic letters under the $A \beta$ pathway. Anti-amyloid agents are also shown in solid white letters above the therapeutic targets in the A pathway.

Oxidative Damage Is the Earliest Event in Alzheimer Disease. Our observations indicate that increased oxidative damage is an early event in $\mathrm{AD}$ that decreases with disease progression and lesion formation. These findings suggest that $\mathrm{AD}$ is associated with compensatory changes that reduce damage from reactive oxygen. The activities and expression of a number of antioxidant enzymes such as $\mathrm{Cu} / \mathrm{Zn}$ - and Mn-superoxide dismutase, glutathione peroxidase, glutathione reductase, and catalase have been studied in AD and could be in part responsible for the decrease in oxidative damage we observed.

\section{Imaging of the $\mathrm{AD}$ disease}

"Clinical criteria for the diagnosis of AD include dementia established by clinical examination and neuropsychological testing, defi- cits in two or more locations of cognition, progressive worsening of memory and other cognitive functions, no disturbance in consciousness, onset between ages 40 and 90 , and absence of systemic disorders or other brain disease to account for the progressive cognitive decline. A diagnostic laboratory test for $\mathrm{AD}$ has not been found and $\mathrm{AD}$ remains a diagnosis of exclusion. A definitive diagnosis cannot be made without neuropathological confirmation.

Two neuropathological criteria are available for the diagnosis of AD. The major microscopic alterations in AD are SP and NFT formation, selective neuron loss and shrinkage, synapse loss, neuropil thread formation, and amyloid antipathy". Decrease in glutathione is also a major event that is associate with neurodegenerative (Alzheimer's, Parkinson's, as examples) diseases. Most antioxidant defenses (SOD, GSH-PX, a-tocopherol) do not seem to be substantially changed in the aging brain, but glutathione (GSH). concentration and the glutathione redox index are lowered.

\section{GSH as a biomarker for AD}

Various lines of evidence indicate that the brain operating system (oxidative stress) is a fundamental factor underlying AD etiology. GSH levels have consistently been shown to reflect operating system status. Furthermore, the literature reviewed so far reveals a strong correlation between AD pathology and reduced GSH levels. These findings spurred the development of tests for GSH levels as a biomarker for AD. Several methodologies have been developed to assess GSH levels in peripheral biological samples, such as blood. Recent advances in technology have also enabled non-invasive in vivo measurement of GSH directly in various brain regions that use MRS. We discuss recent findings from studies using different GSH measurement methodologies and evaluate their relative potential to serve as a reliable measure of GSH levels.

It was postulated that either b-amyloids or Glutathione may serve as biomarkers in the diagnosis of the neurodegenerative diseases with $\mathrm{AD}$ as priority. Polyphenols and resveratrol and curcumin were known for years to be agents for the repairing of dementia. Gazit has identified a curcumin- $\beta$-amiloid complex and published the crystal structure of a congo red-amyloid complex (Figure 7).

Furthermore, Cole and co workers had demonstrated that curcumin stains hippocampus sections, thus concluding that like Congo red and thioflavin S, curcumin binding is not sequence-dependent but rather conformation- dependent. 

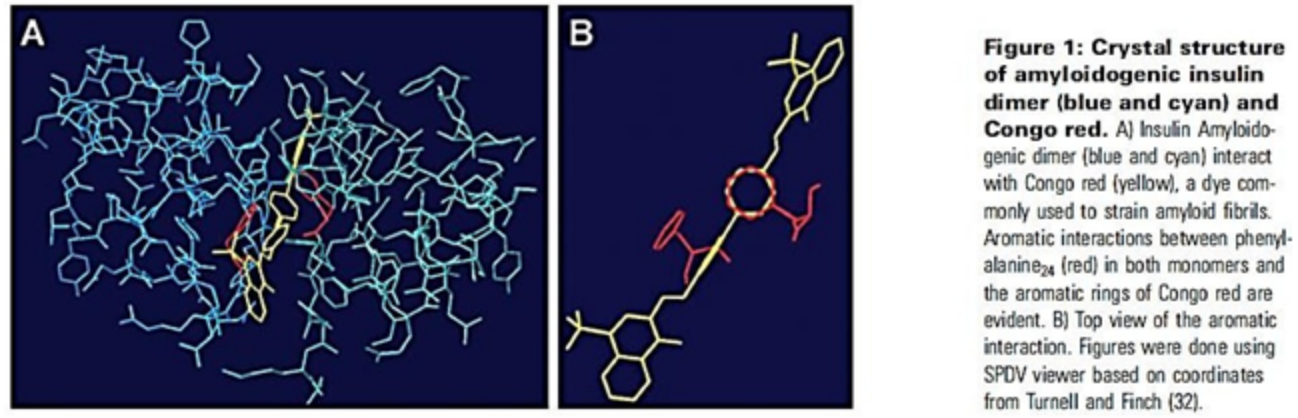

Figure 7

As for glutathione, its production in the hippocampus is reduced when oxidative stress takes place at the early stages of $\mathrm{AD}$. It is reported that use of bimanes can aid in the detection of glutathione that is excreted from the brain. In sections from rat brain GPx (GPx, glutathione peroxidases;) immunoreactivity has been found predominantly in neurons in cortex, hippocampus and cerebellum. Glutathione in living organisms can be identified due to the transfer of Bimane with the catalysis of Glutathione S-Transferases.

The detection of the bimanyl-glutathion adduct is carried out as follows: Confocal microscopy was carried out using an inverted confocal microscope (Zeiss LSM 510) with a water-immersion633 objective lens. The $364 \mathrm{~nm}$ line of the argon UV laser (Coherent, Inc.) was used to excite the MCB-GSH adduct, and fluorescence was collected between 440 and $490 \mathrm{~nm}$ to construct the MCB image. A $488 \mathrm{~nm}$ laser line was used to excite calcein, and the image was acquired between 505 and $550 \mathrm{~nm}$. Again, data were digitized to 12 bits. The pinhole of the confocal was set to acquire optical slices of; $1 \mathrm{~mm}$ for all images.

\section{Glutathione in the AD}

Transcription of GSH peroxidase and GSSG reductase was elevated in hippocampus and inferior parietal lobule, but not in cerebellum of $\mathrm{AD}$ patients, which may reflect the protective gene response to the increased peroxidation in the brain regions showing severe AD pathology. The levels of glutathione transferase, a protective enzyme against aldehydes and especially HNE were decreased in the brain and ventricular CSF of autopsied AD and normal control subjects. Together these data imply that oxidative stress plays an important role in the pathogenic process but that alterations in the glutathione system are secondary to other events leading to neurodegeneration.

Congo Red (CR) and Thioflavin T (ThT) have afforded the starting point for the visualization of amyloid plaques and are still commonly employed in post mortem histological analyses However, due to their charge these probes are unsuitable for in vivo applications]. The development of small radiolabeled molecules for use as biological markers of $\beta$-amyloid $(A \beta)$ deposits in Alzheimer's disease (AD) has been a goal of researchers for several years. due to their charge these probes are unsuitable for in vivo applications. To address this issue, several laboratories developed probes with noncharged, lipophilic and low-molecular weight chemical structures (MW < 650) that facilitate crossing of the blood-brain barrier.

The use of lipophilic reagents was preferers based on their better penetration through the BBB. However, The Agent are of high molecular weight and therefore their effectivity in the staining of the peptides in the inner brain is limited. Two research groups have prepared imaging agents that are of low $(<650)$ molecular weight and could penetrate the BBB. The design was based on a carrier moiety that could pull the polar fluorescent units through the endothelial based BBB.

Texas red is a red-purple fluorescent dye with a relatively low (MW 615) molecular weight. It is to the staining of cells. The agent does not cross the BBB. 
In contrast, Use of Thioflavin derivative resolve individual $\mathrm{A} \beta$ plaques and cerebrovascular amyloid in living microscopy. Future studies will include imaging amyloid load in transgenic mice us- ing newly developed high-resolution micro PET, a technology that will provide a direct transition to PET imaging studies in human subjects.

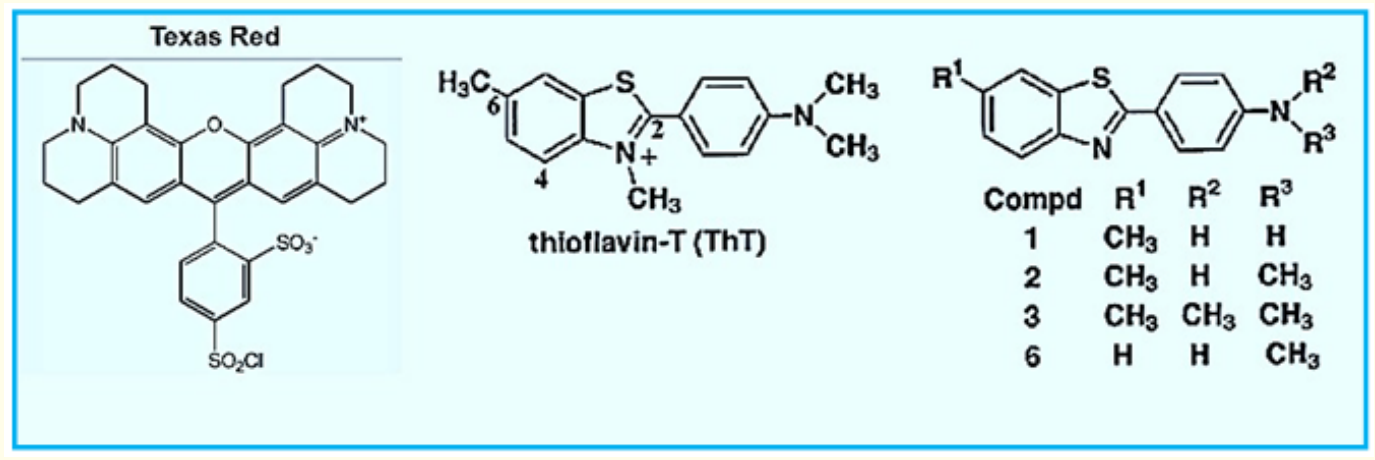

Figure 8

Today, two agents (see below) that might be useful as probes to detect the biomarkers of neurodegenerative diseases were prepared by two groups. Recently, such small molecules that could be supplied to the brain via the blood streams (abdominal or tail in- jection) have been reported by an Israeli and a Korean groups and introduced to the inner brain by crossing the blood brain barrier (BBB).

A typical commercial reagent for amyloid staining is "Amylo -Glo".

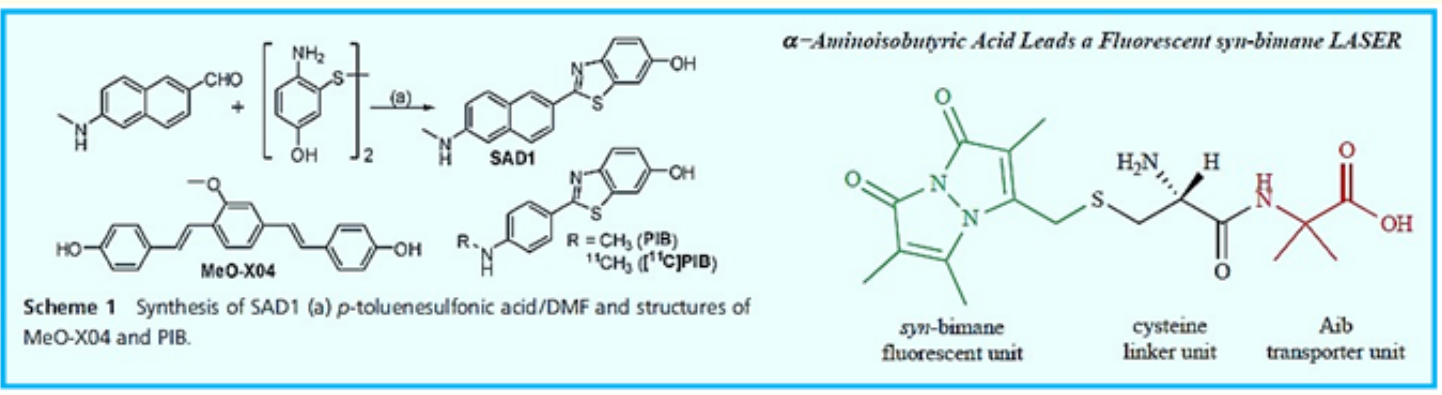

Figure 9

It is still a great challenge to use either biomarker, b-amyloids or Glutathione or other that are produced in the brain, probably in the hippocampus gland in the early events of the neurodegenerative disease (Figure 10).
The green branches of an astrocyte, one of several kinds of glial cells, surrounded by blue nuclei of other cells (credit: karin pierre, institut de physiologie, unil, lausanne. via wikimedia commons). 


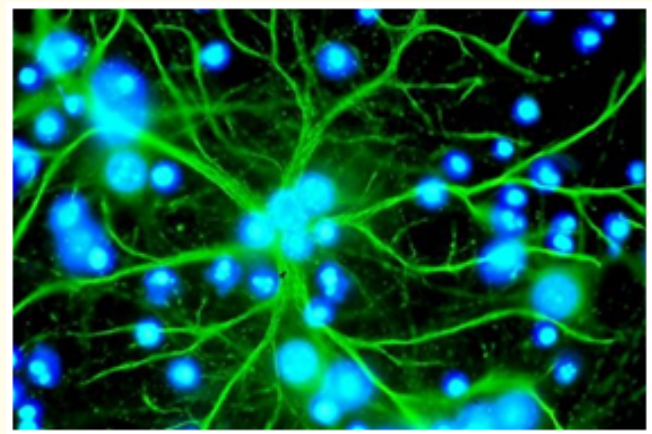

Figure 10

i.e. associated with Alzheimer's disease (AD) is the prominent extract of extracellular amyloid plaques within the anterior brain. These panels are mainly composed of fibrous aggregates of A-beta peptide. Traditional methods for histologic localization of these panels usually rely on the use of Congo red or thioblbin followers. This study describes the characterization of a new fluorescent histochemical probe, Amylo-Glo, for high resolution and contrast localization of amyloid plaques in brain tissue segments. Potential advantages over conventional amyloid plaque stains such as Congo Red or Thioplavin S can be attributed to its unique chemical and spectral properties. Specifically, the result is a very bright blue excited stain under physiological conditions that will not bleed when illuminated with other filters. Its clarity makes it ideal for low-magnification quantification studies, with a unique excitation/emission profile and labeling studies. Light staining conditions make it ideal for multiple immunofluorescent.

3 research tools for analyzing Amyloid peptide in Alzheimer's Disease (AD). The unique properties of the Amylo-Glo ${ }^{\circledR}$, Styrylbenzene derivative, fluorescent substrate which makes Amyloid plaque staining "better, easier and faster".

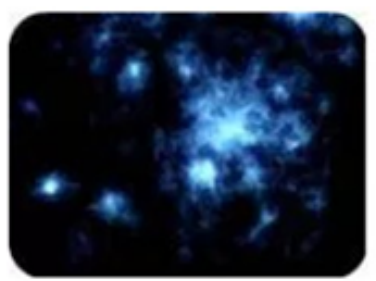

Picture 1: High magnification view of Amylo-Glo labeled amyloid plaque from the cortex of an 11 month old $A D / T g$ mouse with UV illumination only. Note the high clarity, ultra low background and very fine detail available only with Biosensis Amylo-Glo staining reagent.

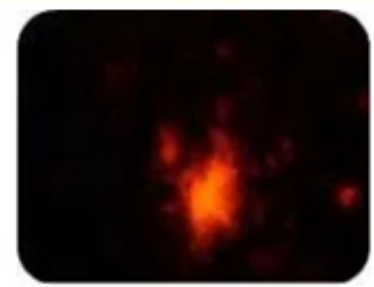

Picture 2: Congo Red stain of amyloid plaque from the cortex of an 11 month old $A D / T g$ mouse with green light excitation. Note that while the central region of the plaque appears uniformly bright as well as some of the large fibrils, the fine details remain obscure. Also the intensity of the fluorescent emission is the weakest of all the tracers

leading to early photo bleaching and making quantification and counting very difficult.

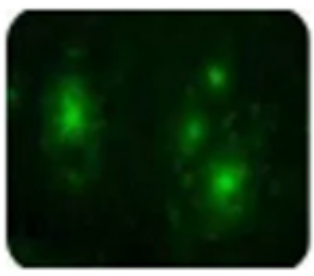

Picture 3: Thioflavin S staining of amyloid plaque from the cortex of an 11 month old $A D / T g$ mouse with blue light excitation. Note that while the central region of the plaque is uniformly stained, the fibrils are not; they are diffuse and cloudy. Also the background staining is relatively high, even showing some relatively faint non-specific cellular labeling as well, leading to a much more difficult plaque quantification and clarity determination

Figure 11 
The only test that can completely diagnose Alzheimer's is a post-mortem brain biopsy. An Alzheimer's diagnosis is given when eliminated everything else that can cause symptoms. Because there is no complete test for Alzheimer's, the strongest diagnosis that can be given is "reasonable Alzheimer's." If most test results are appropriate, but not all, a "possible Alzheimer's" diagnosis can be made. Sometimes doctors may feel that the symptoms do not fit any of the more common dementia, including Alzheimer's, and may give a diagnosis of "NOS dementia (not otherwise specified)".

Most of the instrumental diagnostics on neurodegenerative diseases is carried out on, CSF (Cerebrospinal fluid (CSF). In spite of enormous efforts that were dedicated for a more advanced technique, For this purpose, mainly two methods are available.

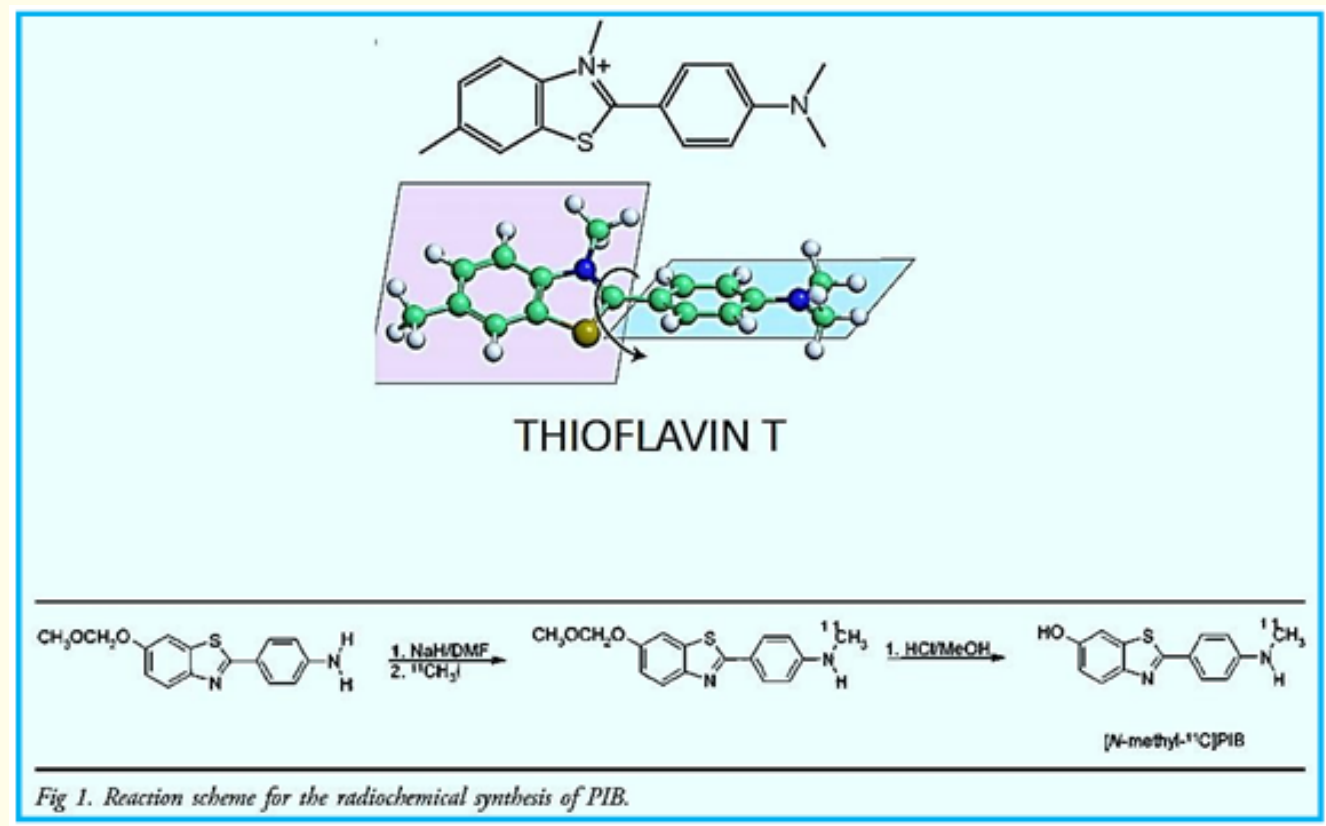

Figure 12

Since its first description in 1959, the fluorescent dye Thioflavin-T (ThT) has become among the most widely used "gold standards" for selectively staining and identifying amyloid fibrils both in vivo and in vitro. The large enhancement of its fluorescence emission upon binding to fibrils makes ThT a particularly powerful and convenient tool. Despite its widespread use in clinical and basic science applications, the molecular mechanism for the ability of ThT to recognize diverse types of amyloid fibrils and for the dye's characteristic fluorescence has only begun to be elucidated.

ThT is among the most widely used amyloid dye among the hundreds of amyloid reports published yearly. The intensive use of ThT as an in vitro marker of amyloid formation has spawned substantial research into the mechanism of ThT binding, with the main goal of answering the following questions:
- What common structure(s) does ThT recognize and how?

- How do these interactions lead to the dramatic increase in ThT fluorescence?

Within the past several years, a series of critical experimental analyses of ThT have illuminated many of the detailed and atomic-level interactions necessary for ThT binding and fluorescence. Because several recent reports have discussed the broad range of amyloid staining dyes, here we focus on recent atomic-level studies of the fibril-binding mechanism of ThT. Through a combination of biophysical and biochemical analysis, protein engineering, fluorescence microscopy, and computational simulation, a coherent mechanism for ThT-fibril interactions has emerged. 


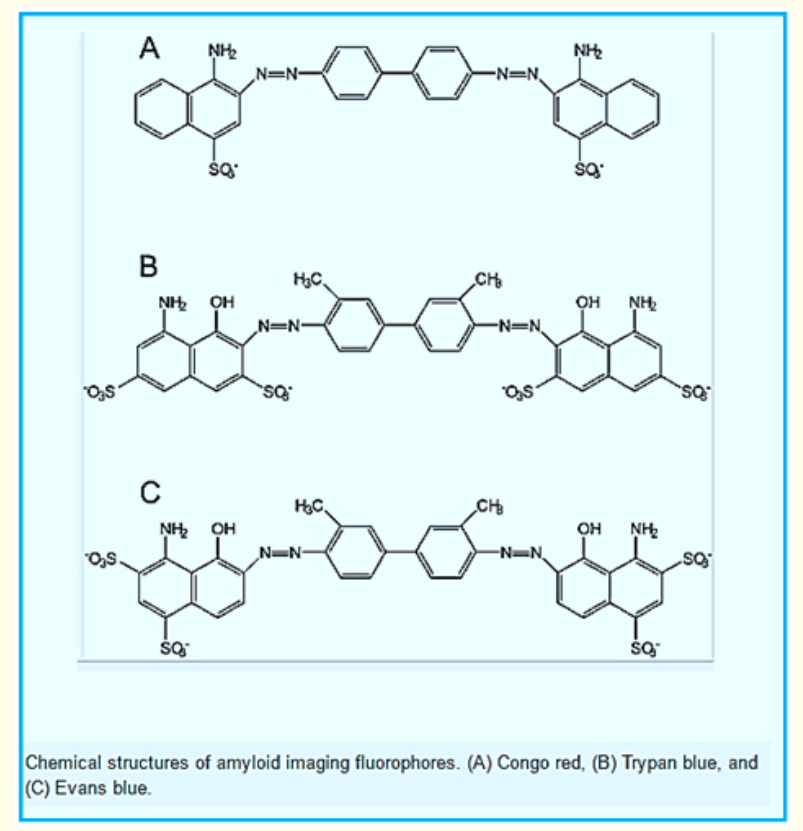

Figure 13
Some Useful staining agents for A- $\beta$ plaques.

Generally, proteins are modified in the organism through oxidative stress in the organism.

Oxidative modification of proteins by reactive oxygen species (ROS) or reactive nitrogen species (RNS) is implicated in the pathogenesis of both normal aging and neurodegenerative diseases. The generation of ROS and RNS may occur by many physiological $l$ and nonphysiologically processes.

One of the most used agents for Neurodegenerative diseases diagnostics is Pittsburgh compound B (PiB) (Figure 14):

It is a radioactive analog of thioflavin $\mathrm{T}$, which can be used in positron emission tomography scans to image beta-amyloid plaques in neuronal tissue. Due to this property, Pittsburgh compound B may be used in investigational studies of Alzheimer's disease.

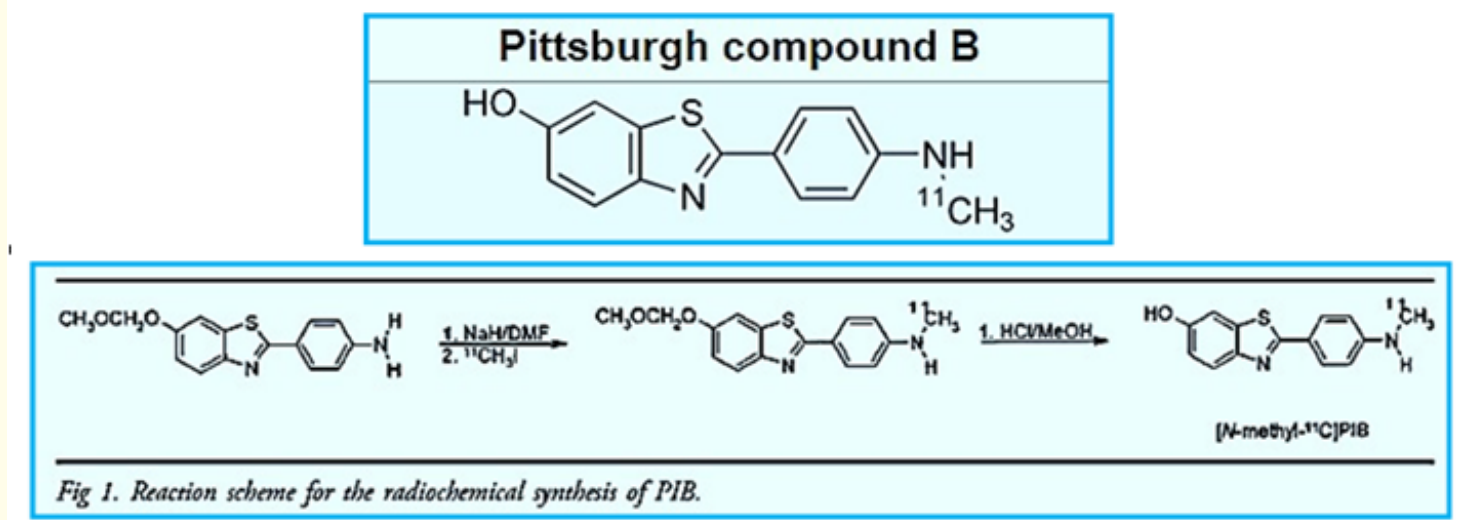

Figure 14

With regard to the amyloid imaging agents, the focus of this review will be on the most widely evaluated positron emission tomography (PET) tracer, Pittsburgh Compound-B (PiB). There is insufficient published evidence available to evaluate any of these F-18 tracers for their potential use in drug trials at present. While the findings discussed for PiB PET may ultimately be found to extend to these F-18-labeled tracers as well, this can not be taken in considerations until appropriate studies have been repeated with each individual tracer or until pharmacological equivalency to $\mathrm{PiB}$ has been established by direct comparison in the same subjects. 
Increasing data over the last years about the patho-mechanisms involved in $\mathrm{AD}$ allow for the development of specific treatment strategies aimed at slowing down or even preventing neuronal death in AD. However, this requires also that

- $\quad \mathrm{AD}$ can be diagnosed with high accuracy, because non-AD dementias would not benefit from an AD-specific treatment;

- $\quad \mathrm{AD}$ can be diagnosed in very early stages when any intervention would be most effective; and

- $\quad$ Treatment efficacy can be reliably and meaningfully monitored. Although there currently is no ideal biomarker that would fulfill all these requirements, there is increasing evidence that a combination of currently existing neuroimaging and cerebrospinal fluid (CSF) and blood biomarkers can provide important complementary information and thus contribute to a more accurate and earlier diagnosis of AD.

The need for early detection of AD is high, however, practically only a few biomarkers that may serve as basis for a suitable diagnostic method are available i.e. Amyloid beta. Glutathione are on the head of the list. Intensive genetic research has identified several potential susceptibility genes for this form of AD, e.g., Apo- $\varepsilon 4$, $\alpha 2$-macroglobulin low- density lipoprotein receptor-related protein, insulin degrading enzyme, and glutathione-S- transferase, but, until now, only Apo- $\varepsilon 4$ has been established firmly as a susceptibility gene. However, only about $50 \%$ of the late-onset AD cases are homo- or heterozygous for Apo- $\varepsilon 4$, and thus its use as diagnostic biomarker for AD is limited. Taken together, although there is evidence of an important genetic component in $\mathrm{AD}$, most $\mathrm{AD}$ is probably caused by complex interactions between one or more susceptibility genes and different environmental factors.

Therefore, it is unlikely that genetic markers can take on a major role as a diagnostic biomarker for AD. However, it can be expected that a better.

A publication in 2011 in "Nature Neuroscience" revealed that Japanese researchers at RIKEN, Japan's flagship research institute, Develop a new breakthrough watery reagent that really makes the biological tissue transparent. Experiments using fluorescence microscopy on reagent-treated samples have been published, producing vivid 3D images of neurons and blood vessels at mouse brain depth. The reactor is very efficient and inexpensive to manufacture, and provides an ideal means of organ analysis and complex networks that sustain living systems
Scientists at Stanford University published in 2013 on the development of the imaging system "Clarity". The method is best represented in the article's abstract in "Nature".

"Obtaining high-resolution information from a complex system, while maintaining the global perspective needed to understand system functioning, is a major challenge in biology. Here we are addressing this challenge in a method (known as CLARITY) to transform whole tissue into a nanoporous hydrogel-hybridized form (linked to a 3D network). Hydrophilic polymers) which is fully complex but optically transparent and permeable to the macrolole. Using mouse brains, we show intact-tissue imaging of long-range projections, local circuit wiring, cellular relationships, subcellular structures, protein complexes, nucleic acids and neurotransmitters. CLARITY also enables intact-tissue in situ hybridization, immunohistochemistry with multiple rounds of staining and de-staining in non-sectioned tissue, and antibody labelling throughout the intact adult mouse brain. Finally, we show that CLARITY enables fine structural analysis of clinical samples, including non-sectioned human tissue from a neuropsychiatric-disease setting, establishing a path for the transmutation of human tissue into a stable, intact and accessible form suitable for probing structural and molecular underpinnings of physiological function and disease".

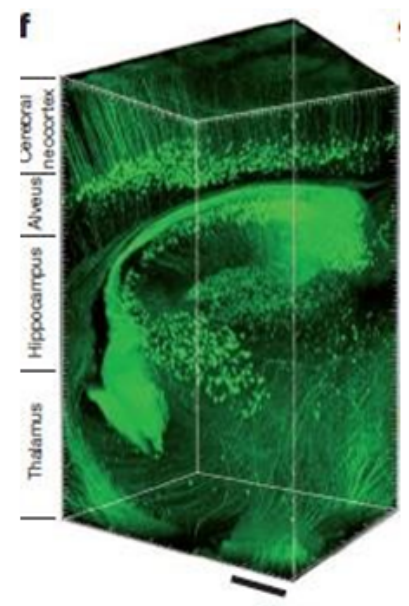

Figure 15

Non-sectioned mouse brain tissue showing cortex, hippocampus and thalamus (310 objective; stack size, 3,400 mm; step size, 2 $\mathrm{mm})$. Scale bar, $400 \mathrm{~mm}$. 
The high resolution and Imaging to very fine brain details is stunning. However, this implies the sacrificing of the mouse.

A study by University of Rochester Scientists in the journal Science reveals that the brain's unique method of waste removal -dubbed the glymphatic system -- Very active during sleep, and clears toxins responsible for Alzheimer's disease and other neurological disorders. Furthermore, the researchers found that during sleep, brain cells reduce their size, allowing waste to be removed more effectively. This revelation may change scientists' understanding of the biological purpose of sleep and point to new ways to treat neurological disorders. Nederdaard found that while asleep, the living brain excretes composed of many effluents ("Brain Garbage") back through the BBB into the blood stream and then to the processing organs, liver, kidneys for excreting out of the organisms. The scientists and coworkers applied some imaging agents for their two photon spectroscopic measurements in their work.

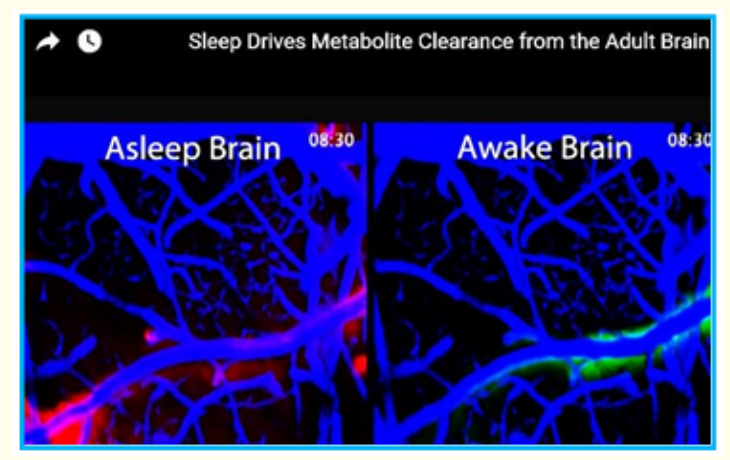

Figure 16

The internet is overflowing from announcements like " New blood test predicts Alzheimer's, dementia" which teaches us that there is a public intensive demand to clarify the Air regarding the neurodegenerative diseases. A doctors-office type of diagnostic method is needed also for drug research towards finding a cure for the disease. Without such aid, drug designers and pharmaceutical research is "walking in the Dark". Today, after more than a century of intensive scientific efforts, we are bouncing between the Amyloid hypothesis and the oxidative stress model. There is not Clearview over the events that take place in the early stages of the $\mathrm{AD}$. Unfortunately, Aging must endure the development of the neurodegenerative diseases and very many individual perish, die due to lack of remedy. The scientist watch the situation and unfortunately cannot do a lot to improve it.

\section{Photoacoustic imaging}

Photoacoustic imaging (PAI) hasbecomeamajortoolforpreclinicalstudiesbecauseofitsuniquefeatureof combining optical and ul- trasound imaging. Its potential for in vitro and in vivo imaging has been demonstrated in both tomographic and photo acoustic microscopy systems. The photoacoustic imaging research network in biology and medicine represents an interdisciplinary consortium of photoacoustic imaging experts in Austria.

It combinesallareasofresearchthatarecurrentlyconsideredtoplayasignificantrole in photoacoustic imaging, namely biology, mathematics, medicine and physics. The main goal is to direct the technique to wards practical applications and routines for biomedical applications.

\section{Conclusion}

The imaging of the inner brain is currently practiced in many clinics based on positron tomography. This procedure uses an in most cases an isotopes laboratory to prepare on site $18^{\mathrm{F}}$ glucose to administer via the blood stream and BBB penetration the $18^{\mathrm{F}}$ glucose which is the energy fuel for the brain. There are only few fluorescent agents with low molecular weight that can be employed. Recently, such small molecules that could be supplied to the brain via the blood streams have been reported by an Israeli and a Korean groups.

Japanese and American scientists have shown that brains could be made transparent and substructures of the inner brain could be identified in detail. Nedergaard investigated the bidirectional fluxes of agents and their metabolites from the brain while asleep. Those extrusions are accumulated in the blood stream and further elaborated to extrusions in the normal metabolic manner.

All these findings can lead to novel diagnostics and treatments of the living and operating brain.

\section{Assets from publication with us}

- Prompt Acknowledgement after receiving the article

- Thorough Double blinded peer review

- Rapid Publication

- Issue of Publication Certificate

- High visibility of your Published work

Website: www.actascientific.com/

Submit Article: www.actascientific.com/submission.php

Email us: editor@actascientific.com

Contact us: +919182824667 